\title{
Presenting a hybrid method in order to predict the2009 pandemic influenza A (H1N1)
}

\author{
Reza Boostani ${ }^{1}$, Mojtaba Rismanchi ${ }^{\mathrm{b}}$,Abbas Khosravani ${ }^{1}$, Lida Rashidi ${ }^{1}$, \\ Samaneh Kouchaki ${ }^{1}$,Payam Peymani ${ }^{2}$,Seyed Taghi Heydari ${ }^{2}$, B. Sabayan ${ }^{2}$, K. B. \\ Lankarani $^{2}$ \\ ${ }^{1}$ CSE\&IT Dept., Faculty of Electrical and Computer Engineering, Shiraz University, \\ Shiraz, Iran \\ boostanieshirazu.ac.ir \\ ${ }^{2}$ Health Policy Research Center, Shiraz University of Medical Sciences, Shiraz, Iran \\ Lankaran@sums.ac.ir
}

\begin{abstract}
:
By the emergence and rapid spread of 2009 pandemic influenza A (H1N1) virus through the world, several methods have been developed to predict and prevent this lethal disease. Although many efforts have been made by statistical and traditional intelligent methods to anticipate this disease, but none of them could satisfy the expectations of specialists. This paper aims to present an efficient hybrid method to predict H1N1 with a reliable sensitivity. In this way, three methods including Gaussian mixture model (GMM), neural network (NN), and fuzzy rule-based system (FRBS) have been fused in order to provide an accurate and reliable prediction scheme to anticipate presence of H1N1influenza. In this study, 230 individuals were participated and their clinical data were collected. The proposed hybrid scheme was implicated and the results showed to be superior to using each of the decision components containing NN, FRBS, and GMM classifiers. The achieved results produced $95.83 \%$ sensitivity and $80.95 \%$ specificity on unseen data which support the effectiveness of the hybrid method to predict the influenza in its golden time.
\end{abstract}

\section{Keywords:}

GMM, Neural Network, FRBC, H1N1 influenza, predictio

\section{Introduction}

Few months after announcement of world health organization (WHO), regarding the start of phase 6 pandemic of swine flu on 11 June 2009, we have witnessed the rapid progression of the first pandemic of twenty first century (2). Since introduction of swine flu (H1N1) infection different reports have been released indicating that $\mathrm{H} 1 \mathrm{~N} 1$ behavior has some unique features including: high rate of hospitalization and mortality, involvement of young age group rather than extreme of ages and higher risk of influenza complications among patients with morbid obesity, that differentiate it from seasonal flu (1). Furthermore, the magnitude of hospitalization and mortality rate and to top it off imposed burden on health care system and affected patients showed a substantial difference in comparison with seasonal flu (3). In this setting the adverse impact of this pandemic have not limited to the health care systems while a great social fear in the large gatherings and travelers (e.g. among pilgrims) was among consequences of this event (4).

DOI : 10.5121/acij.2012.3103 
Due to unusual and atypical clinical and laboratory presentations of H1N1 influenza with its consequent difficulties in differentiation of swine flu from seasonal one and even common cold, late or misdiagnosing have led to some complications for the affected patients (5). To avoid delaying in proper decision making and rational prescription of antiviral medication, oseltamivir, this study aims to develop a hybrid model based on fuzzy, statistical and neural learning theories in order to predict presence of this infection in its vital period. The proposed hybrid scheme benefits all strong points of human-like decision making (fuzzy method), generalization and robustness of statistical models and adopt the ability of human-like neural learning to gather all these capabilities in one hybrid model. The rest of this paper is structured as follows. First, the related works are explained and their positive and negative points are discussed. Next, the methodology has been used in this article is presented. Afterward, experimental results are brought and analyzed in the results and discussions section. Finally, the paper is concluded by a conclusion and future works part.

\section{Related Works}

Prediction of lethal diseases has attracted many attentions and the number of research teams who are working on this issue is significantly increasing(6-8). Several the state-of-art methods have been employed in this field such as polynomial predictors (23), support vector machines (SVMs) (24), fuzzy predictors such as adaptive neuro-fuzzy interference system (ANFIS) (10), as well as neural approachesincluding multi-layer perceptron(MLP), radial basis functions (RBFs) and modular neural networks [11].

A variety of medical tasks has been successfully performed using such methods including detection of electrocardiogram (ECG) arrhythmia using fuzzy classifier (9), decision-making in pathology by neural networks $(17,25)$, prediction of influenza vaccination (26), presenting a stochastic model to predict pandemic influenza(27), prediction of gastro-intestinal absorption using adaptive splines(28), prediction of pulmonary embolism (29) by neural network, and early detection in mammographic images by SVMs and fuzzy SVMs (30-31). Moreover, the mentioned prediction methods were used commonly in other fields such as predicting of stock market indexes (32) by SVM, forecasting of weather status by neural networks (33), predicting on sea waves behaviors to anticipate Tsunamis using neural network(34), prediction of Alzheimer prognosis based on estimating the electroencephalogram (EEG) behaviors (35), forecasting the seizure attack time for epileptic patients (36), and prediction the cancer growth(37).Although lots of research has been done for preparation of societies to inhibit the spreading of influenza A (H1N1) but no reliable prediction method has been proposed which is verified FDA institute. Therefore, this paper is aimed at predicting H1N1 influenza using quantitative clinical signs and symptoms in its golden time. To achieve this aim, this study presents a hybrid model based on statistical methods as well as neural network and fuzzy logic to predict the influenzaA (H1N1) in its golden time.

\section{Materials and methods}

\section{Subjects and source of information}

Based on the national protocol in Islamic Republic of Iran (12-13), a suspected case of influenza A (H1N1) virus infection was defined as one who has high grade fever $\left(>38^{\circ} \mathrm{C}\right)$ or has at least two acute respiratory symptoms including: nasal obstruction/rhino rhea, sore throat, cough, fever (feverishness) and met at least one of the following criteria:

1) Within the last seven days returned from a country or region with an epidemic of influenza A (H1N1). 
2) Being in close contact (within two meters) with a confirmed case of influenza A (H1N1) within the past seven days.

3) Patients with moderate to severe respiratory illness requiring hospitalization, or unexplained or unusual clinical patterns associated with serious or fatal cases.

On the other hand confirmed case of influenza A (H1N1) was defined as one who has high grade fever $\left(>38^{\circ} \mathrm{C}\right)$ or has at least two acute respiratory symptoms including: nasal obstruction/ rhino rhea, sore throat, cough, fever (feverishness) and influenza A (H1N1) virus infection that has been confirmed by Reverse Transcriptase PCR (RT- PCR). RT- PCR is the gold standard test to rule in or rule out the presence of virus and for this reason we provided the information for training of the software from confirmed cases and suspected cases for those the result of RT- PCR became negative.

\section{Artificial neural network}

Artificial neural networks (11)have been developed for a wide variety of problems such asclassification, function approximation, and prediction. A neural networkis structured by a parallel full-connection computation units arranged in layers mimicking the physiologic structure of the brain. There are multiple connections within and between the layers whichindicate the strengths or weights between neuronsthat are learned under an optimization criterion. All Information learnt by the network is stored in the interconnection weights between each two successive layers. In medical research, the most commonly used artificial neural networks (ANN) are multi-layer perceptrons (MLP) which schematically depicted in Fig. 1. The implemented neural network uses back propagation in order to learn the parameters (weights) of the model, usually squared error or maximum likelihood, using a gradient optimization method (11). In MLP networks, the error (the difference between the predicted and the target values) is propagated back from the output to the connection weights and updates the weightsto minimize the prediction error (11). The employed MLP is constructed by threelayerswith back propagation learning algorithm. Quantitative clinical symptoms of are used as input features to the network and the training algorithm iteratively modified the numeric values of these weights to decrease the training error of the network. In order to avoid over-fitting, 10-times 10-folds cross validation is utilized to determine the optimum parameters of the MLP in terms of number of neurons in the hidden layer (here is selected 6) and the function type of neurons (which is selected as sigmoid). After training of the MLP, the weights are fixed for the test phase. When a set of input feature values are presented to the trained network, an output is generated to predict the label of the input vector. By applying a sign function the predicted value is classified to the positive or negative classes which show that the patient is with or without the H1N1, respectively. It should be noted that there is no overlap between the train and test data and in other words, test set is considered as blind or unseen data.

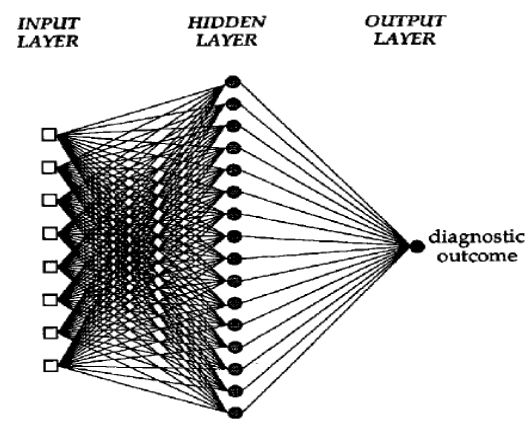

Figure 1: Architecture of an MLP neural network with one hidden layer is schematically shown 


\section{Gaussian mixture model (GMM)}

Statistical models have always been applied to prediction of different diseases. One of the models which are repeatedly being utilized for influenza prediction is Gaussian mixture model (GMM) (14,22). GMM models the probability density function of the observed data using a multivariate Gaussian mixture density. This mixture is enabledto estimate any distribution with arbitrary shape. GMM is formulated in terms of Gaussian functions and their importance. The mentioned components are expressed as follows.

$$
\begin{gathered}
F(x ; \theta)=\sum_{i=1}^{K} p_{i} g\left(x ; m_{i}, \sigma_{i}\right) \\
g\left(x ; m_{i}, \sigma_{i}\right)=\frac{1}{\left(\sqrt{2 \pi} \sigma_{i}\right)^{D}} e^{-\frac{1}{2}\left(\frac{\left\|x-m_{i}\right\|}{\sigma_{i}}\right)^{2}}
\end{gathered}
$$

where $p_{i}$ is the weight of $i$ 'th Gaussian function. A graphic representation of GMM is depicted in Fig. 2.In the current problem, we are faced with a two-class problem; therefore, a GMM should be trained to recognize the patterns of each class separately. First we apply $K$-means to clustering input data of each class separately. $K$-means tries to find those clusters in which data are distributed. The point is that $K$-means is not a stable algorithm due to this fact that the formed clusters depend seriously on the initial centers considered in each run. To improve stability of $K$-means, we have applied a method to initialize the cluster centers such that the outcome will be more stable [15]. In this approach the cluster centers have the maximum distance from each other.

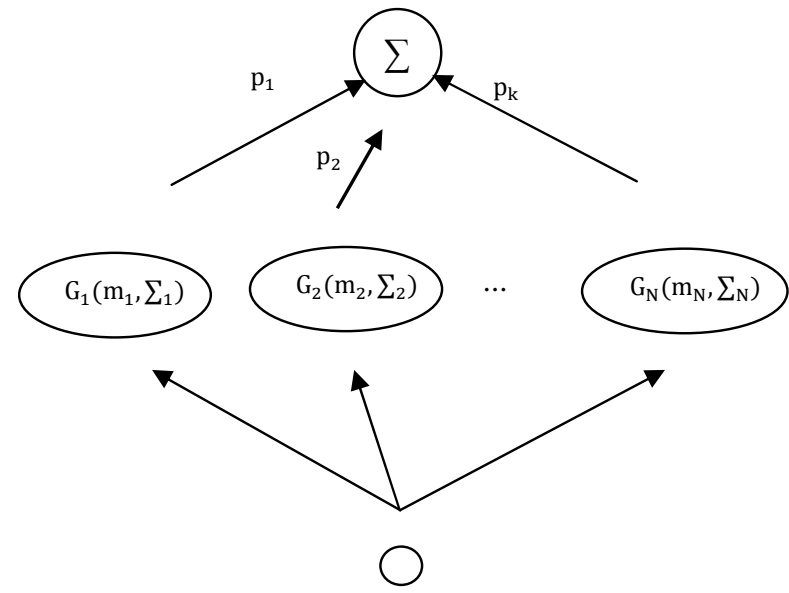

Figure2: Gaussian mixture model consisting of Gaussian functions along their importance

Another problem in $K$-means is the number of clusters which should be known beforehand. There are several methods for finding the number of clusters among which Gap-Statistic has proven to be quite applicable [16]. In this algorithm the optimum number of clusters is found. Therefore, it is expected that the formed clusterswould be denser and are placed far away from each other.

After finding the initial clusters, we apply expectation maximization (EM) algorithm to find the Gaussian components for each of the two classes.

EM is a soft version of $K$-means algorithm in which the data membership in each cluster is a binary value ( 0 or 1 )while in $\mathrm{EM}$, an observation can have a membership degree ranged from 0 
to 1. It means data can belong to more than one Gaussian component with different probabilities. The clusters' centers and number of instances in each cluster are used as Gaussian mixture model parameters.

Finally the probability of data is calculated in each class and the division is compared to a threshold;consequently,the class label can be determined. Afterward, the model is trained and assessed by 10 -times 10 -folds cross validation in order to avoid over-fitting. Regarding the training results, a threshold is determined for each GMM model. Next, the trained GMM is applied to the test data and the weighted summation of Gaussian outputs for each input pattern determines its probability given the model. Finally, to take the last decision, each pattern is applied simultaneously to the trained models, and a simple maximizing operand determines the predicted label. In order to use GMM as a predictor instead of a classifier, the maximum GMM real value is considered as the predicted value with regard to that input sample.

\section{Fuzzy rule-basedclassifier}

One of the efficient learning methods which mimic human-like decision, especially in disease diagnosis, is fuzzy rule-based system(FRBS) which is a special case of fuzzy modeling where the output of the system is crisp and discrete. The main advantage of this classifier is the interpretability and human understandability of the model [6].Basically, to train a FRBS, a compact set of fuzzy If-Then rules should be found in order to model the input-output behavior of the system (20).

Rules are generated from a set of labeled data from all classes(19). After the training phase, we can test the classifier with unseen patterns. Many approaches have been proposed to construct a rule engine from input numerical data. These include heuristic approaches $(7,9)$,neuro-fuzzy techniques (11), and clustering methods(8).Here, we use fuzzy rules of the following type for this problem:

Rule $R_{j}$ : If $x_{1}$ is $A_{j l}$ and $\ldots$ and $x_{n}$ is $A_{j n}$ then $C$ with $C F_{j}=a_{j}, j=1,2, \ldots, N$,

where $\mathrm{X}=\left[\mathrm{x}_{1}, \mathrm{x}_{2}, \ldots, \mathrm{x}_{\mathrm{n}}\right]$ is the input feature vector, $A_{j k}$ is the fuzzy set associated to $x_{k}, C$ is the class label, $C F_{j}$ is the certainty degree of rule $R_{j}$ and $N$ is the number of fuzzy rules in the rule-base system.In order to classify an input pattern $X_{t}=\left[x_{t 1}, x_{t 2} \ldots x_{t n}\right]$, the degree of compatibility of that pattern with each rule should be computed (i.e., using a T-norm to model the "and" connectives in the rule antecedent). In case of using product asT-norm, the compatibility grade of rule $R_{j}$ with the input pattern $X_{t}$ can be calculated as:

$$
\mu_{j}\left(X_{t}\right)=\prod_{i=1}^{n} \mu_{i} \cdot A_{j i}\left(x_{t i}\right) .
$$

Where $\mu_{j}\left(X_{t}\right)$ is the fuzzy membership value of its input pattern $X_{t}$ in the $j$ 'th membership function. In case of using weighted vote $(10,20,21)$ as the reasoning mechanism, each fuzzy rule gives a vote for its consequent class.

$$
\begin{aligned}
& \mu+=\sum_{\begin{array}{l}
\mu j(X t)>C F j \\
\text { and } j=1, \ldots, N
\end{array}} \mu j(X t) \\
& \mu-=\sum_{\mu j(X t)<C F j} \mu j(X t) \\
& \quad \text { and } j=1, \ldots, N \\
& \text { 1. If } \mu+>\mu \text { - then } X_{t} \text { is classified as positive. } \\
& \text { 2. If } \mu+<\mu \text { - then } X_{t} \text { is classified as negative. } \\
& \text { 3. If } \mu+=\mu \text { - then don't classify } X_{t} .
\end{aligned}
$$

Twostages for generating rules are used in this study as shown in Fig.3. In the first stage, rules are generated for each training data. Triangular fuzzy sets are used and number of fuzzy sets 
Advanced Computing: An International Journal ( ACIJ ), Vol.3, No.1, January 2012

and parameters of membership functions (MFs) on attributes are dependent to values of that attribute. We also determine degree of each rule and select rules with maximum degrees. Degrees are determined based on compatibility of training data with fuzzy sets defined on each attribute as described in $(13,18)$. In the second stage, the objective function is constructed in terms of sensitivity and specificity (i.e. simulated annealing) to improve the FRBS performance. Finally, a majority vote mechanism is used to determine the class label.

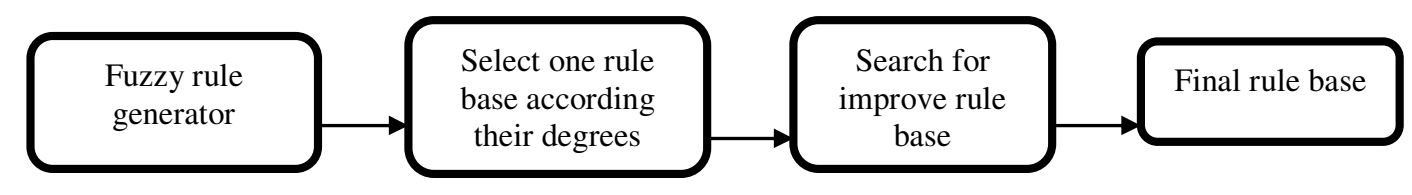

Figure 3: stages of constructing of an FRBS

\section{The proposed hybrid method}

We proposed a method composed of three components in terms of artificial Neural Network (ANN), fuzzy rule-basedclassifier (FRBS), and Gaussian mixture model (GMM). In the proposed method, the decision is made based on the result of each of the three methods. A weighted voting mechanism is used and a class with themajorvote is selected to determine the label of the input data. We first trained each of the above methods independently using the training data to find their proper parameters. Finally a hybrid method is constructed using the three methods and weights of each method are adjusted using the training data as shown in Fig.4.

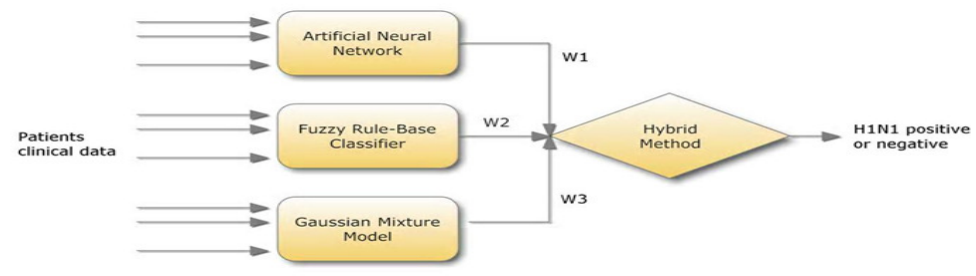

Figure 4: Structure of the proposed hybrid model using MLP, FRBS and GMM modalities are shown

\section{Results and Discussions}

In this section, the achieved results by each of the modalities along with the hybrid method are presented. In this study, 230 cases were selected randomly from the examination results of patients at Namazi and Aliasghar hospitals which were under supervision of Shiraz University of Medical Sciences. The diagnoses were performed by professional specialists from 15 September to 10 December 2009. A list of clinical attributes and their corresponding positive percent used in this experience is shown in Table 1. 
Advanced Computing: An International Journal ( ACIJ ), Vol.3, No.1, January 2012

Table 1: Items belonged to patients' history, signs and symptoms

\begin{tabular}{|c|c|}
\hline Symptom & Percent of positive \\
\hline Age & - \\
\hline Occupation & - \\
\hline Travel history & $14 \%$ \\
\hline Cough & $84.96 \%$ \\
\hline $\begin{array}{l}\text { History of } \\
\text { exposure }\end{array}$ & $32.74 \%$ \\
\hline Headache & $20.80 \%$ \\
\hline Dizziness & $6.19 \%$ \\
\hline Chills & $26.99 \%$ \\
\hline Sore throat & $29.65 \%$ \\
\hline Dyspnea & $47.35 \%$ \\
\hline $\begin{array}{c}\text { Rhino rhea or } \\
\text { Nasal congestion }\end{array}$ & $4.87 \%$ \\
\hline Body pain & $73.89 \%$ \\
\hline $\begin{array}{l}\text { Nausea or } \\
\text { Vomiting }\end{array}$ & $39.82 \%$ \\
\hline Diarrhea & $14.60 \%$ \\
\hline Convulsion & $0.44 \%$ \\
\hline Chest pain & $10.62 \%$ \\
\hline Abdominal pain & $17.26 \%$ \\
\hline Temperature & - \\
\hline $\begin{array}{l}\text { Respiratory } \\
\text { distress }\end{array}$ & $19.47 \%$ \\
\hline $\begin{array}{c}\text { Abnormal } \\
\text { auscultatory sound }\end{array}$ & $1.77 \%$ \\
\hline Dehydration & $0.88 \%$ \\
\hline Decreased level & $0.88 \%$ \\
\hline $\begin{array}{l}\text { Anemia requiring } \\
\text { transfusion }\end{array}$ & $4.87 \%$ \\
\hline $\begin{array}{c}\text { Pandemic } \\
\text { influenza vaccine }\end{array}$ & $1.77 \%$ \\
\hline Asthma & $7.96 \%$ \\
\hline Obesity & $1.33 \%$ \\
\hline $\begin{array}{c}\text { Other chronic lung } \\
\text { diseases }\end{array}$ & $0.44 \%$ \\
\hline Pregnancy & $5.31 \%$ \\
\hline $\begin{array}{l}\text { Congestive heart } \\
\text { failure }\end{array}$ & $0.44 \%$ \\
\hline Transplant & $0.88 \%$ \\
\hline $\begin{array}{l}\text { Chronic renal } \\
\text { failure }\end{array}$ & $0.88 \%$ \\
\hline $\begin{array}{l}\text { Immune } \\
\text { Suppressive } \\
\text { Condition }\end{array}$ & $1.33 \%$ \\
\hline Active cancer & $0.44 \%$ \\
\hline
\end{tabular}


We used polymerase chain reaction (PCR) test results as the label for each case. PCR tests showed that $37 \%$ of patients were infected by H1N1. We trained the proposed method using 10-times 10-folds cross validation to evaluate the effectiveness of our method. The results achieved by applying each decision making component was separately determined. Then, the hybrid method was employed and the results are compared to those achieved by each modality. A comparison of the performance of each method is shown in Table 2. Different terminology is used to show the performance of each method including sensitivity or true positive rate (TPR) which measures the proportion of actual positives which are correctly identified as such; specificityor true negative rate (TNR) which measures the proportion of actual negatives which are correctly identified. Moreover,other indexes such as positive predictive value (PPV) which measures the proportion of the identified positives that are actually positive, negative predictive value (NPV) which measures the proportion of the identified negatives which are actually negative and finally the accuracy which measures the proportion of all cases which are identified correctly are calculated using applying the data to the models. Another significant criterion, especially in the medical applications, is area under curve (AUC) which is a nonparametric measure of discrimination. The receiver operating characteristic (ROC) area measures the relative goodness of the predictions entirely by comparing the predicted probability of each patient with that of all the other patients. Receiver operating characteristic area is independent of both the prior probability of each prediction and the threshold cut-off for classification. In addition, the mean and standard deviation for each terminology is also reported in Table 2. It can be seen that the hybrid method outperformed the other modalities in terms of TPR, NPV, accuracy, and AUC indexes.

Table 2: Comparison of the performance of each method

\begin{tabular}{lllllll}
\hline & TPR & TNR & PPV & NPV & Accuracy & AUC \\
\hline ANN & $90 \pm 5.92$ & $77.14 \pm 8.11$ & $69.95 \pm 7.34$ & $93.38 \pm 4.93$ & $81.81 \pm 5.25$ & $90.05 \pm 4.36$ \\
FRBC & $91.37 \pm 6.72$ & $69.44 \pm 10.58$ & $63.03 \pm 7.44$ & $94.19 \pm 4.51$ & $77.27 \pm 5.75$ & $80 \pm 4.82$ \\
GMM & $80.55 \pm 9.08$ & $\mathbf{8 6 . 6 1} \pm \mathbf{9 . 6 9}$ & $\mathbf{7 9 . 7 0} \pm \mathbf{1 2 . 0 3}$ & $88.56 \pm 3.72$ & $84.09 \pm 4.21$ & $83.15 \pm 3.28$ \\
Hybrid & $\mathbf{9 5 . 8 3} \pm \mathbf{6 . 4 5}$ & $80.95 \pm 3.69$ & $75.91 \pm 5.66$ & $\mathbf{9 7 . 2 2} \pm \mathbf{4 . 3 0}$ & $\mathbf{8 6 . 3 6} \pm \mathbf{4 . 0 7}$ & $\mathbf{9 2 . 5 2 \pm 4 . 5 3}$ \\
\hline
\end{tabular}

To test for significant difference betweenthe proposed hybrid method and its decision making components,the statistical T-test is utilized to reveal the significance of our results. The statistics t-test allows us to determine $p$-value that indicates how likely we could have gotten these results by chance. If the $p$-value is less than 0.05 , we can conclude that there is a statistically reliable difference between the mean results of the two methods. The $p$-value result of T-test for each terminology between the hybrid method and ANN, FRBS, and GMM methods is reported is Table 3. We are able to test whether the significant improvements of the hybrid method in prediction has enough generalization across the unseen data (test set) or not. T-test reveals that the hybrid method predictions of unseen patients were significantly more accurate than each of the FRBC, MLP, and GMM methods separately.

Our hybrid method exploits its components and compensate for each method's disadvantages. Neural networkderives its computing power through its massively parallel distributed structure and its ability to learn and generalize. The nonlinearity, adaptability, confidence in decision making and neurobiological analogy of neural network makes it a powerful tool for a reliable prediction. Fuzzy inference systems can incorporate and handle uncertainty; therefore,it 
Advanced Computing: An International Journal ( ACIJ ), Vol.3, No.1, January 2012

provides a systematic calculus to deal with it linguistically, and it performs numerical computation by using linguistic labels specified by membership functions. A selection of fuzzy if-then rule can effectively model human expertise but it suffersadequate adaptability to deal with changing environment. Gaussian mixture model is useful in case of multi modal distributions but is an unstable method due to its high sensitivity to itsinitialization values. In order to have a method that benefits all advantages of each described modality, a hybrid scheme is proposed which fuse the results of different modality and takes its decision according to the majority vote scheme.

In order to make our work applicable in hospitals, we designed a user-friendly graphic user interface (GUI) software to predict H1N1 influenza by each modality or using hybrid method. The GUI of this software allows the user to select one of the four models which is shown in Fig. 6. This software is now being used is many hospitals which are all under supervision of Shiraz University of Medical Sciences to assist low-experienced graduated doctors to detect H1N1 influenza.

Table 3: T-test for significant difference between Hybrid method and each of the ANN, Fuzzy and GMM method

\begin{tabular}{|c|c|c|c|c|c|c|c|c|c|c|c|c|}
\hline & & & & & & & TPR & TNR & PPV & NPV & $\begin{array}{c}\text { Accura } \\
\text { cy }\end{array}$ & $\begin{array}{c}\mathrm{AU} \\
\mathrm{C}\end{array}$ \\
\hline & \multicolumn{6}{|c|}{ ANN } & 0.049 & 0.19 & 0.058 & 0.07 & 0.044 & 0.2 \\
\hline & \multicolumn{4}{|c|}{ Fuzzy } & & & 0.147 & $\begin{array}{c}0.00 \\
4\end{array}$ & $\begin{array}{c}0.000 \\
4\end{array}$ & $\begin{array}{c}0.14 \\
1\end{array}$ & $\begin{array}{c}0.000 \\
9\end{array}$ & 0 \\
\hline GM & 0.000 & 0.10 & 0.38 & 0.00 & 0.23 & 0 & & & & & & \\
\hline $\mathrm{M}$ & 3 & 9 & 3 & 01 & 6 & 0 & & & & & & \\
\hline
\end{tabular}




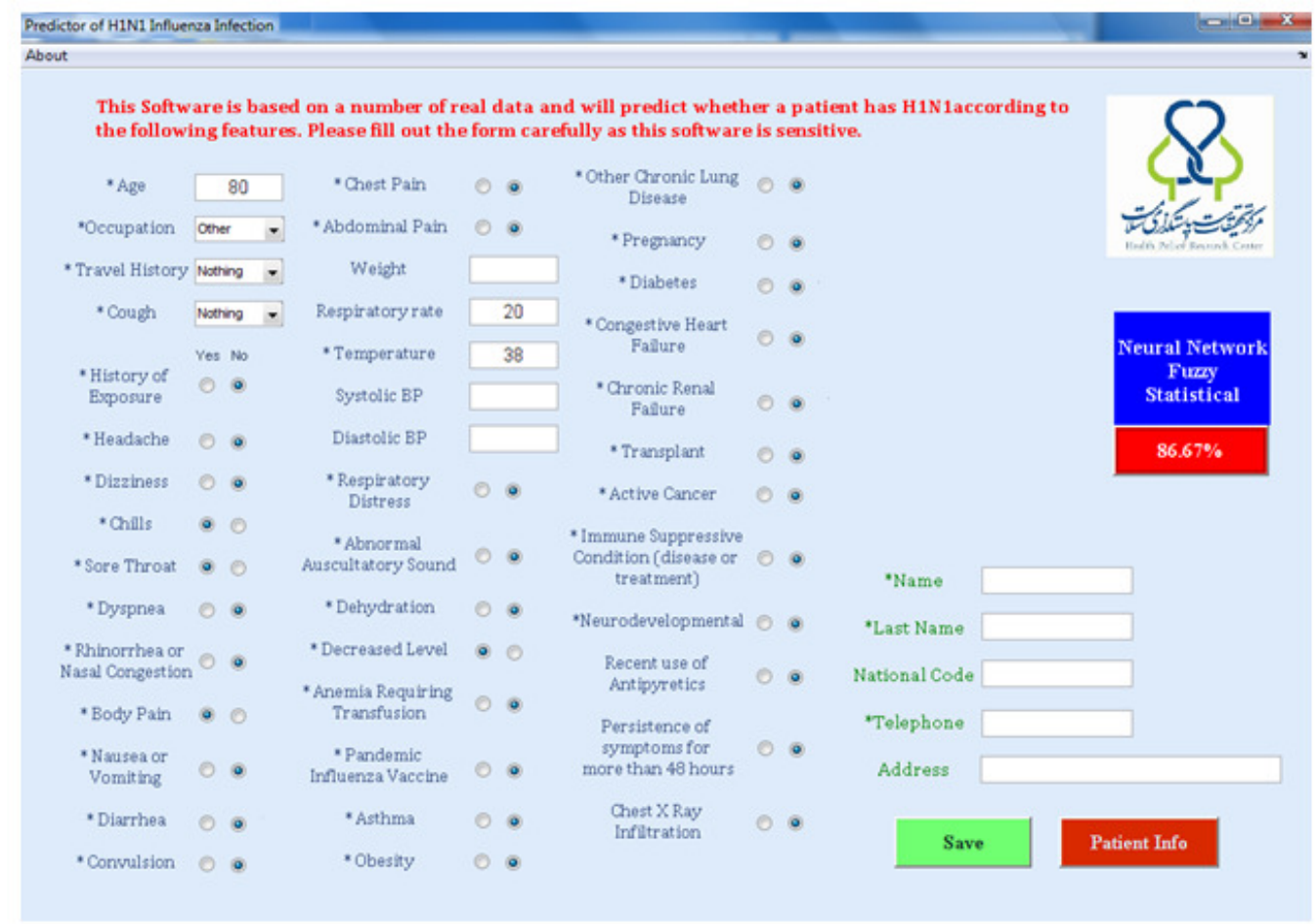

\section{Conclusion and Future Works}

In this paper we proposed a hybrid method to predict H1N1 influenza with high sensitivity and specificity that can satisfy the specialist expectations. The proposed method is able to learn well, takes a robust decision and models the statistics of input data. The reason of inheriting such strong capability is to use three prediction modalities in which each prediction component is the state-of-art for one of the mentioned properties. To compare the hybrid scheme with each of its components (FRBS, MLP, and GMM), the achieved results illustrated in Table 2, exhibits the supremacy of the combined scheme compare to each of its component in terms of TPR, NPV, accuracy, and AUC indexes.

Although the hybrid method has an acceptable performance in this data set, but an adaptation process can refine the model parameters to increase its performance. For instance, the FRBS component can be improved using genetic algorithm to optimize its rule set and also tuning its fuzzy membership functions on each attribute separately [9]. To enhance GMM capability, using another clustering algorithm that is more stable than $k$-means could result in better performance.

Some clinical attributes such as chest X-Ray infiltration were not available; therefore, if we measure more clinical quantitative symptoms like the chest status, for each suspicious subject, the hybrid method is expected to result in a higher accuracy, robustness, and generalization properties. 
Advanced Computing: An International Journal ( ACIJ ), Vol.3, No.1, January 2012

\section{References}

[1] Gooya MM, Soroush M, Mokhtari-Azad T, Haghdoost AA, Hemati P, Moghadami M, Sabayan B, Heydari ST, EmamiRazavi SH, Lankarani KB, "Influenza A (H1N1) pandemic in Iran: report of first confirmed cases from June to November 2009”, Arch Iran Med. 2010;13(2):91-8.

[2] Jain S, Kamimoto L, Bramley AM, et al. "Hospitalized patients with 2009 H1N1 influenza in the United States", April-June 2009. N Engl J Med 2009;361:1935-44.

[3] Viboud C, Miller M, Olson D, Osterholm M, Simonsen L, Preliminary Estimates of Mortality and Years of Life Lost Associated with the 2009 A/H1N1 Pandemic in the US and Comparison with Past Influenza Seasons, PLoSCurr Influenza. 2010 Mar 20:1153.

[4] K. B. Lankarani, Hajj and Swine Flu Pandemic (H1N1, 2009): What Is Expected and What Should Be Done? International journal of Iranian red crescent society, 2010, 12(1), 4-6.

[5] Complications of seasonal and pandemic influenza. Rothberg MB, Haessler SD. Crit Care Med. 2010 Apr;38(4 Suppl):e91-7.

[6] Marta Kolasa, RyszardWojtyna, RafałDługosz, and WojciechJóźwicki, Application of Artificial Neural Network to Predict Survival Time for Patients with Bladder Cancer, Computers in Medical Activity, 2009, 65, 113-122.

[7] Andrew S. Jones, Azzam G. F. Taktak, Timothy R. Helliwell, John E. Fenton, Martin A. Birchall, David J. Husband, An artificial neural network improves prediction of observed survival in patients with laryngeal squamous carcinoma, European Archives of Oto-Rhino-Laryngology, 2006(263), 541-547..

[8] Marchevsky AM, Patel S, Wiley KJ, Stephenson MA, Gondo M, Brov RW, Artificial neural networks and logistic regression as tools for prediction of survival in patients with Stages I and II non-smoker cell lung cancer. Mod Pathol 1998, 11:618-625.

[9] S Osowski, TH Linh, ECG beat recognition using fuzzy hybrid neural network, IEEE Transactions on Biomedical Engineering, 2001, 48(11), 1265-1272.

[10] Jyh-Shing Roger Jang, Chuen-Tsai Sun, EijiMizotani, Neuro-fuzzy soft computing : a computational approach to learning and machine intelligence, Matlab curriculum series, Prentice Hall, 1997.

[11] Simon Haykin, "Neural Network: A comprehensive foundation”, 2nd Edition, 1999.

[12] Moghadami M, kazeroni A, Honarvar B, et al. Influenza A (H1N1) Virus Pandemic in Fars Province: A Report from Southern Iran, July-December 2009. IRCMJ 2010; 12(3):231-238.

[13] Gooya MM, Soroush M, Mokhtari-Azad T, et al. Influenza A (H1N1) pandemic in Iran: report of first confirmed cases from June to November 2009. Arch Iran Med 2010;13(2):91-8.

[14] F. Segovia, J.M. Górriz, J. Ramírez, D. Salas-González, I. Álvarez, M. López, R. Chaves, P. Padilla, Classification of functional brain images using a GMM-based multi-variate approach, Neuroscience Letters, 2010, 474(1), 58-62.

[15] Mohammad Taheri, Reza Boostani, Novel auxiliary techniques in clustering, International Conf. on computer science and engineering (London), July, 2007.

[16] Robert Tibshirani, Guenther Walther, Trevor Hastie, Estimating the number of clusters in a data set via the gap statistic, JRSS: Series-B, 2001(63), 411-423.

[17] Kemal Polat, SalihGüneş, Prediction of hepatitis disease based on principal component analysis and artificial immune recognition system Applied Mathematics and Computation, 2007, 189(2), 12821291.

[18] R. Mikut, J. Jakel, and L. Groll, "Interpretability issues in data-based learning of fuzzy systems", Fuzzy Sets Syst., 2005 (150), 179-197.

[19] S. Abe, M.S. Lan, A method for fuzzy rules extraction directly from numerical data and its application to pattern classification, IEEE Trans. On Fuzzy Systems, 1995, 3(1), 18-28. 
Advanced Computing: An International Journal ( ACIJ ), Vol.3, No.1, January 2012

[20] H. Ishibuchi, T. Nakashima, T. Morisawa, Voting in fuzzy rule-based systems for pattern classification problems, Fuzzy Sets and Systems, 1999, 103(2), 223-238.

[21] J. Casillas, O. Cordon, F. Herrera, Improving the Wang and Mendel's Fuzzy Rule Learning Method by Inducing Cooperation Among Rules, 2001.

[22] Douglas A. Reynolds, Richard C Rose, Robust Text-Independent Speaker Identification Using Gaussian Mixture Speaker Models, IEEE Trans. On Speech and Audio processing, 1995, 3(1), 7284.

[23] A.P. Wilczynski, Use of tensorial polynomial strength function for strength prediction in laminated polymeric composites, Composites Science and Technology, 1994, 51(4), 525-530.

[24] VeladimirVapnic, The nature of statistical learning theory, Springer-Verlag, 2nd Edition, 1999.

[25] Robert L. Becker, Computer-assisted image classification: use of neural networks in anatomic pathology, Cancer Letters, 1994, 77(2-3), 111-117.

[26] LjiljanaTrtica-Majnaric, MarijanaZekic-Susac, NatasaSarlija, Branko Vitale, Prediction of influenza vaccination outcome by neural networks and logistic regression, Journal of Biomedical Informatics, In Press, Corrected Proof, Available online 6 May 2010.

[27] AntonellaLunelli, Andrea Pugliese, Caterina Rizzo, Epidemic patch models applied to pandemic influenza: Contact matrix, stochasticity, robustness of predictions, Mathematical Biosciences, 2009, 220(1), 24-33.

[28] E. Deconinck, Q.S. Xu, R. Put, D. Coomans, D.L. Massart, Y. Vander Heyden, Prediction of gastro-intestinal absorption using multivariate adaptive regression splines, Journal of Pharmaceutical and Biomedical Analysis, 2005, 39(5), 1021-1030.

[29] Isabelle Chagnon, Henri Bounameaux, DrahomirAujesky, Pierre-Marie Roy, Anne-Laurence Gourdier, Jacques Cornuz, Thomas Perneger, Arnaud Perrier, Comparison of two clinical prediction rules and implicit assessment among patients with suspected pulmonary embolism, The American Journal of Medicine, 2002, 113(4), 269-275.

[30] FatemehMoayedi, ZohrehAzimifar, Reza Boostani, SerajodinKatebi, Contourlet-based mammography mass classification using the SVM family, Computers in Biology and Medicine, 2010, 40(4), 373-383.

[31] F. Moayyedi, R. Boostani, A. Kazemi, S. D. Katebi, "Combination Methods of Fuzzy Techniques with SVM to Enhance the Mass Detection in Mammograms", Iranian Journal of Fuzzy Systems, 2010, 7(1), 23-33.

[32] Kyung-Shik Shin, TaikSoo Lee, Hyun-jung Kim, An application of support vector machines in bankruptcy prediction model, Expert Systems with Applications, 2005, 28(1), 127-135.

[33] Guoqiang Zhang, B. Eddy Patuwo, Michael Y. Hu, Forecasting with artificial neural networks: The state of the art, International Journal of Forecasting, 1998, 14(1), 35-62.

[34] Michele Romano, Shie-YuiLiong, Minh Tue Vu, PavloZemskyy, Chi Dung Doan, My Ha Dao, PavelTkalich, Artificial neural network for tsunami forecasting, Journal of Asian Earth Sciences, 2009, 36(1), 29-37.

[35] Ursula Schreiter-Gasser, Theo Gasser, Peter Ziegler, Quantitative EEG analysis in early onset Alzheimer's disease: correlations with severity, clinical characteristics, visual EEG and CCT, Electroencephalography and Clinical Neurophysiology, 1994, 90(4), 267-272.

[36] ArianeSchad, Kaspar Schindler, BjörnSchelter, Thomas Maiwald, Armin Brandt, Jens Timmer, Andreas Schulze-Bonhage, Application of a multivariate seizure detection and prediction method to non-invasive and intracranial long-term EEG recordings, Clinical Neurophysiology, 2008, 119(1), 197-211.

[37] P.P. Delsanto, C.A. Condat, N. Pugno, A.S. Gliozzi, M. Griffa, A multilevel approach to cancer growth modeling, Journal of Theoretical Biology, 2008, 250(1), 16-24. 


\section{Authors}

\section{Short Biography}

Kamran BagheriLankarani was born in 1965 in Tehran. He got his MD. Diploma from Shiraz University of Medical Sciences (SUMS), Shiraz Islamic Republic of Iran on 1989. He entered medical residency in the same year and graduated on 1992.He passed the national board exam on internal medicine in the same year. He later received an award from the President of Islamic Republic of Iran at that time for his performance on this exam. He received his subspecialty board on Gastroenterology and hepatology on 1996. He joined the department of internal medicine of SUMS on 1992 as an assistant

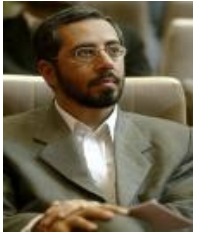
professor. He served as the director general of Nemazi hospital in Shiraz, vice chancellor of SUMS and as the head of department of internal medicine and finally as the minister of health and medical education until September 2009. Currently he is professor of internal medicine at SUMS and a senior member of Gastroenterology and Hepatology Research Center at SUMS, chairman of Health Policy Research Center and a senior staff member of liver transplant ward at SUMS. He is also a member of several national and international professional societies. He has published over 100 papers in different fields of gastroenterology and hepatology. He also was co-author of 5 books in this field. He is the editor-in-chief of Shiraz Electronic Medical Journal and the Iranian Red Crescent Medical Journal. He is also the chairman of hepatitis monthly Journal editorial board and an editorial board member and reviewer of several other national and international journals. 